\title{
Optimizing quality collaboratives
}

\section{Sheila Leatherman}

While the evidence base for the effectiveness of quality collaboratives is emerging, valuable knowledge can be gained from experts in order to design and optimize implementation of collaboratives.

\section{A METHOD GAINING ACCEPTANCE}

"Quality collaborative" is the term used to describe a technique which is increasingly being used in a number of countries and is perceived by participants as a valuable method of sharing experience, accessing expertise, and providing an environment which supports quality improvement endeavours. Although the name is rather generic, the form and functions of quality collaboratives are constantly being refined through real world learning. Regrettably, the published evidence base is not as replete as one would hope when such a resource intensive intervention is enjoying popularity. Anecdotal reporting and insights of experts are therefore important for designing and implementing collaboratives to optimize their effectiveness.

\section{OLD AND NEW CHALLENGES}

In this issue of QSHC Øvretveit and colleagues ${ }^{1}$ present their recommendations as 10 challenges for organizing and implementing collaboratives to maximize import. Many of the problems noted are shared by other quality improvement interventions-in fact, they are endemic to the basic challenges of management. Although seemingly selfevident, we are appropriately reminded that change management is inherently dependent upon clarity of intent, shared goals, explicit definition of resource requirements, and stability of purpose.

A number of the challenges might be most constructively understood as indictments of the state of the art of quality measurement and management, as well as admonitions for realizing the value of collaboratives. Three stand out: (1) data analysis, (2) accountability for achieving results, and (3) sustaining effort. Each of these critical functions requires both will and skill. Firstly, collaboratives face the same difficulties as do many other quality interventionsnamely, that validated data are not readily accessible nor are the requisite analytical skills available in every institutional setting where a collaborative is working. Secondly, ongoing measurement is imperative but is often sacrificed to other pressing duties. Collaboratives need to be held accountable, as do all quality improvement interventions, for the precious resources expended both in money and in human effort. For example, a collaborative focused on cancer care in the UK is reported to involve 10000 individuals and $£ 5$ million of expenditure. A regular account of progress against explicitly agreed objectives should be required. Finally, sustainability requires a diligent effort to institutionalize the change intervention and a commitment to monitoring progress. These three functions are rudimentary to quality management of any sort.

Less generic are the considerations peculiar to effecting change in health services. The challenges noted here may, in fact, represent uniquely valuable contributions which are by-products of implementing quality collaboratives. For example, collaboratives rely on clinical teams and, in many cases, physicians are in key roles. When effectively implemented, the clinician must assume the role of institutional change agent, which is different from the agent of the patient. These new "systems thinking skills" for clinicians are likely to have other secondary benefits. Another strength of collaboratives is the relatively efficient use of experts to facilitate and guide multiple institutional teams to internalize best practice and translate the opportunity to their own setting. This access to expertise may not have been available to individual institutions.

\section{MOVING FORWARD FOR SYSTEMIC IMPROVEMENT}

Over the past two decades there has been an ebb and flow of quality improvement methodologies in health care, but few of these methods have been linked to a published evidence base of effectiveness. Enthusiasm has taken the place of evidence, and we have placed faith in "magical fixes" that fail to meet our expectations, such as the excessive reliance on medical audit or, more recently, the hopes that publicly released performance data will have a dramatic impact on system performance. ${ }^{2}$ It is therefore important to heed the experts' own acknowledgement of the deficient empirical evidence base for quality collaboratives.

We also know, however, that there is a clear logic of and need for simultaneously using a number of levers for change to systemically improve health care. ${ }^{3}$ Such a strategy would selectively use collaboratives as one of many approaches alongside such interventions as payment reform, regulation, incentives, and performance monitoring to effect constructive change. The relative strength of quality collaboratives awaits further definitive research but, in the meantime, we can benefit from listening to the experts who are gaining valuable knowledge in how to design and implement quality collaboratives most effectively.

Qual Saf Health Care 2002;11:307

\section{Author's affiliation}

S Leatherman, School of Public Health, University of North Carolina, USA

Correspondence to: Professor S Leatherman 2211 West 49th Street, Minneapolis, MN 55409, USA; sheilaleatherman@aol.com

\section{REFERENCES}

1 Øvretveit J, Bate P, Cleary P, et al. Quality collaboratives: lessons from research. Qual Saf Health Care 2002;11:345-51.

2 Marshall $M$, Shekelle $P$, Leatherman $S$, et al What do we expect to gain from the public release of performance data? A review of the evidence. JAMA 2000;283: 1866-74.

3 Leatherman S. Applying performance indicators to improve health systems performance. Measuring up: improving health systems performance in OECD countries. Paris: OECD, 2002. 